\title{
UMA VISÃO MODERNA DO ENSINO DO DESENHO TÉCNICO MECÂNICO VIA CAD INTEGRADO À MATEMÁTICA E COMPUTAÇÃO
}

\author{
A MODERN VIEW OF TEACHING MECHANICAL DRAWING USING CAD INTEGRATED TO
} MATHEMATICS AND COMPUTING

Douglas Domingues Bueno ${ }^{1}$, Camila G. Gonsalez Bueno ${ }^{2}$, Rodrigo Borges Santos ${ }^{3}$

DOI: 10.37702/REE2236-0158.v40p255-265.2021

\begin{abstract}
RESUMO
O desenho técnico mecânico é relevante ferramenta para projeto e fabricação de peças e máquinas. Tem seus princípios baseados em geometria descritiva e evoluiu de prática totalmente manual, empregando elementos como papel e lápis, para operações de maior produtividade, envolvendo programas computacionais (CAD, do inglês Computer Aided Design). Embora a elaboração de desenhos técnicos em papel permita o desenvolvimento de importantes habilidades, os educandos em engenharia têm sido levados também a se dedicarem a praticar o uso de interface gráficas de software de CAD. Essa nova realidade cria uma excelente oportunidade de ampliar a formação em desenho técnico, integrando fundamentos de matemática e computação para explicar, de forma ilustrativa, o funcionamento básico de programas computacionais de CAD. Essa visão permite ampliar o aprendizado, de forma complementar, na formação em Engenharia Mecânica. Assim, o presente artigo traz essa reflexão, citando exemplos de como se pode gerar a integração do desenho técnico mecânico com fundamentos de matemática e computação associados aos programas de CAD.
\end{abstract}

Palavras-chave: ensino de desenho técnico; CAD; Integração, Matemática e Computação.

\begin{abstract}
Mechanical drawing is a relevant tool for designing and manufacturing parts and machines. Its principles are based on descriptive geometry, and it has evolved from a totally manual practice, using elements such as paper and pencil, for higher productivity operations involving computer programs (CAD, Computer Aided Design). Although to make technical drawings on paper allows one to develop important skills, engineering students have also been dedicated themselves to practicing the use of graphics user interface of CAD software. This new context creates an excellent opportunity to improve the technical learning of drawing by integrating fundamentals of mathematics and computing. This integration allows one to illustrate the basic functioning of CAD computer programs. In this context, the present article introduces this point of view, showing illustrative examples of how it is possible to generate the integration of mechanical technical drawing with fundamentals of mathematics and computation associated with CAD programs.
\end{abstract}

Keywords: technical drawing learning; CAD; Integration, Mathematics and Computing.

\footnotetext{
1 Professor, Doutor, Universidade Estadual Paulista (UNESP), Faculdade de Engenharia de Ilha Solteira (Feis, UNESP) - Departamento de Matemática; douglas.bueno@unesp.br

2 Pós-doutoranda em Engenharia Mecânica, Doutora, Universidade Estadual Paulista (UNESP), Faculdade de Engenharia de Ilha Solteira (Feis, UNESP); cggonsalez@gmail.com

3 Professor, Doutor, Universidade Federal da Grande Dourados (UFGD), Faculdade de Engenharia - Departamento de Engenharia Mecânica; rodrigobsantos@ufgd.edu.br
} 


\section{INTRODUÇÃO}

Ao longo dos anos o ensino do desenho técnico tem sido parte fundamental na formação de profissionais das áreas de tecnologia, especialmente em cursos de engenharias e arquitetura. Em destaque, o uso de um conjunto de informações técnicas de desenhos nos padrões de normas tem impacto direto em tempo e custo de projetos, principalmente no contexto das atividades de engenheiros mecânicos e civis.

Os cursos clássicos de desenho técnico buscam, em geral, desenvolver no futuro profissional a capacidade de ler e elaborar desenhos, com ênfase na visualização de objetos baseada em conceitos de geometria descritiva. Essencialmente, eles tratam da representação de formas tridimensionais em planos (bidimensionais), utilizando projeções, análises de distâncias lineares e angulares, entre outros fundamentos. Tais desenhos são comumente elaborados à mão, haja vista o importante potencial de desenvolvimento de capacidade de abstração espacial. Embora haja evidências sobre as vantagens dessa abordagem, devido à expressiva evolução de microprocessadores, dos dispositivos de memória e capacidade de armazenamento de dados digitais, as pranchetas têm sido substituídas por computadores.

Nesse contexto, o ensino do desenho técnico tem passado por significativas transformações ao longo dos anos. Programas de CAD/CAM (do inglês, Computer Aided Design e Computer Aided Manufacturing), especialmente para Engenharia Mecânica, têm se popularizado nas versões para representações bi e tridimensionais. Esses recursos têm gerado profundo impacto de produção tecnológica por facilitar análises físico-matemáticas de predição de desempenhos e comportamentos, por auxiliar a fabricação de peças com máquinas automáticas programáveis, além de permitirem simulações digitais de montagens de máquinas e dispositivos antes mesmos dos componentes estarem fisicamente disponíveis. Essa realidade, impulsionada principalmente por demandas da indústria, tem criado um ambiente favorável para modernizar as relações de ensino e aprendizagem e, consequentemente, gerado oportunidade da criação de novas abordagens para o ensino do desenho técnico.

Monnerat (2012) discute o ensino do desenho técnico, no contexto de um curso de arquitetura, utilizando diferentes recursos de CAD ao invés de papel e lápis. A autora menciona ganhos inclusive na percepção de aumento de motivação dos educandos engajados no processo de aprendizagem. Pereira et al. (2011) também discutem uma proposta pedagógica de ensino para um curso de Engenharia de Produção Civil baseada no uso de vídeos, elaboração de maquetes, além de apresentação de seminários. As autoras relatam uma percepção de melhoria da dinâmica do curso de desenho, principalmente como resultado de trabalho em equipe e atividades de planejamento. Silva (2003) também explora recursos computacionais para o ensino do desenho técnico mecânico. $\mathrm{O}$ autor elabora e apresenta um ambiente de hipermídia, baseado em multimídia e hipertexto, e o destaca como facilitador ao desenvolvimento da aprendizagem.

Diversas publicações disponíveis na literatura têm mostrado uma tendência do ensino de desenho técnico utilizando recursos de computação, especialmente para engenharias. Trindade (2002), por exemplo, discute uma proposta de ensino usando internet e hipermídia para construir o aprendizado. A autora explora também conceitos de autoaprendizado auxiliado pelo computador. Anteriormente, Silva (2001) também desenvolveu um ambiente conceitualmente semelhante, compreendendo, principalmente, $o$ estudo de cortes e seções e a busca por um processo adequado para viabilizar a autoaprendizagem. Esses trabalhos, entre outros, apresentam claramente como recursos de CAD podem auxiliar na aprendizagem e no desenvolvimento da inteligência espacial (GARDNER, 2011).

Embora seja praticamente indiscutível a existência de vantagens no uso de programas de CAD, especialmente no contexto das atividades de projeto e produção industrial, nota-se que não são encontradas significativas evidências 
de que seus fundamentos têm sido abordados nos cursos de desenho técnico de forma ampla. Essa observação pode ser confirmada nos livros básicos de desenho técnico assistido por computador que, invariavelmente, apresentam parte introdutória para desenho à mão e, na sequência, detalhes de configuração e uso de programa $\mathrm{CAD}$, com especificações de normas técnicas para peças, conjuntos, dispositivos e edificações. Assim, com tal abordagem de ensino, para o educando, potencialmente não há explícita correlação dos recursos de comandos de CAD (como traçar uma linha perpendicular a um plano, girar um objeto em torno de um ponto, entre outros) com os fundamentos matemáticos e computacionais necessários para a execução da tarefa.

Destaca-se que a relevância do aprendizado mencionado, apresentando fundamentos empregados em programa CAD, é particularmente maior para cursos de graduação em engenharia com disciplinas de desenho nos primeiros semestres, pois nesse período introdutório, em geral, há em tais cursos uma concentração de disciplinas de matemática como Cálculo Diferencial e Integral, Álgebra Linear, Geometria Analítica, entre outras. Então, tem-se uma oportunidade de ampliar o ensino de desenho técnico através da integração entre prática de desenhos em CAD e matemática e computação. Assim, nesse contexto, o presente trabalho apresenta uma visão dessa integração, que permite potencializar o ensino do desenho técnico para engenharias, agregando conhecimento de tópicos discutidos em disciplinas típicas de cursos de engenharia, especialmente envolvendo operações básicas de álgebra linear e geometria analítica, permitindo maior compreensão dos fundamentos de software de CAD e ampliando a formação em desenho técnico.

\section{O ENSINO DO DESENHO TÉCNICO}

Os cursos de desenho técnico mecânico tipicamente compreendem aprendizados teóricos e práticos, que em geral compreendem três principais aspectos: i) apresentação de simbologias, geralmente especificadas em normas; ii) leitura e interpretação de desenhos; e iii) elaboração de desenhos. Esses conteúdos podem ser distribuídos durante o ensino, de tal modo que o aluno não necessariamente note a separação de forma explícita. O fluxograma apresentado na Figura 1 ilustra essas ideias.

Figura 1 - Fluxograma de uma estrutura típica de curso de desenho técnico mecânico para engenharia

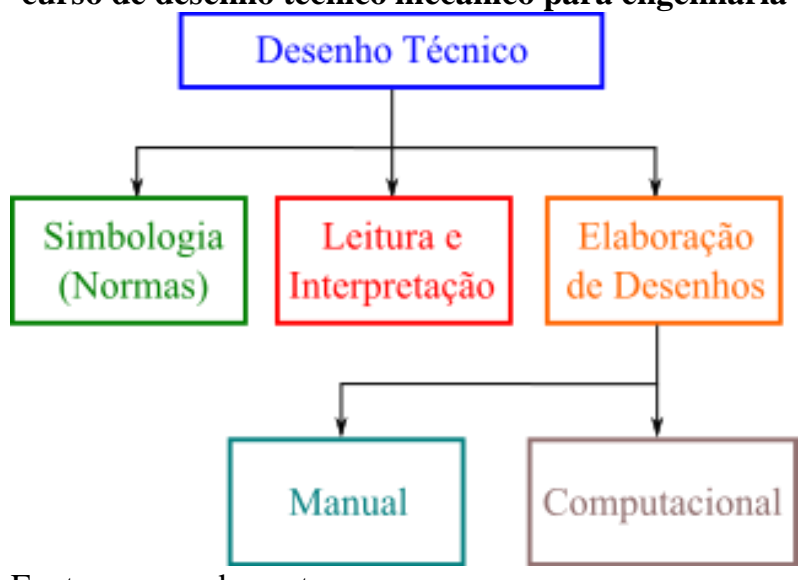

Fonte: acervo dos autores.

Nos aspectos ligados à prática do desenho técnico, tem-se que a elaboração de desenhos comumente envolve execução de forma manual ou computacional, sendo eventualmente consideradas ambas as possibilidades durante $o$ curso. Sabe-se que a execução manual permite desenvolver habilidades de processos de desenhos, especialmente por envolver conceitos de geometria e capacidade de abstração. Também, emprega-se materiais clássicos, como lápis, papel e borracha, além de instrumentos tradicionais do desenho, como régua, esquadros e compassos, entre outros.

Além da execução de desenhos manuais, tem-se atualmente como prática comum a elaboração utilizando software CAD. No entanto, a disponibilidade e variedade de programas computacionais de $\mathrm{CAD}$, aliadas à acessibilidade a computadores pessoais, pode favorecer uma prática superficial, especialmente devido a dois fatores. $\mathrm{O}$ primeiro é o fato de que os softwares de CAD, seguindo uma tendência geral das áreas de computação, têm apresentado modernas interfaces gráficas, exigindo cada vez menos dos usuários, visto que em sua maioria são desenvolvidos para aplicações comerciais, que naturalmente visam a facilidade de uso. Como segundo fator, tem- 
se que a prática pode se limitar à execução de desenhos, propriamente dita, sem demandar que o usuário se atente para os fundamentos de matemática e computação empregados no programa computacional, especialmente devido à alta disponibilidade de informações como videoaulas gratuitas em plataformas da internet, manuais de usuário em formato digital e acessível, entre outros aspectos, que induzem essa interação em menor grau.

Diante de tal contexto apresenta-se uma reflexão que a integração da execução de desenho usando CAD com aspectos básicos de matemática e computação podem potencializar o ensino do desenho técnico mecânico, adicionando relevante conteúdo aos educandos. Essa visão é ilustrada na Figura 2, que destaca o estrito uso de software como apenas uma limitada parte ao se comparar com todo aprendizado que pode ser gerado ao se integrar à execução de desenhos técnicos os fundamentos de matemática e computação. Para clareza de entendimento da visão apresentada neste artigo, mostra-se na próxima seção alguns exemplos da integração proposta.

Figura 2 - Visão ilustrativa do potencial aprendizado ao se integrar fundamentos do CAD ao estrito uso de sua interface gráfica

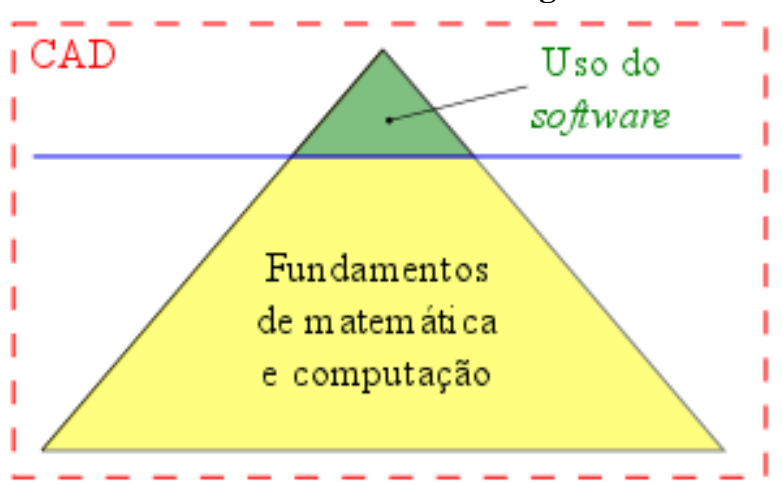

Fonte: acervo dos autores.

\section{INTEGRAÇÃO À MATEMÁTICA E COMPUTAÇÃO}

Para ilustrar a reflexão introduzida neste artigo, a seguir são apresentados exemplos de recursos comumente disponíveis nos programas de CAD com uma fundamentação matemática para ampliar o aprendizado do desenho. Destaca-se que não se pretende indicar estratégias de implementação computacional de qualquer programa de $\mathrm{CAD}$ em particular, mas sim sugerir uma base matemática para realizar as operações que são demonstradas nos exemplos. Ainda, entende-se que uma operação disponível em um comando de programa de CAD pode ser implementada computacionalmente por mais de uma forma. No entanto, os resultados apresentados a seguir podem ser compreendidos sem perda de generalidade.

\section{Rotacionar figuras planas}

A rotação de figuras planas é um recurso típico nos programas de CAD. Em geral, um comando de rotação permite fixar um ponto do plano em torno do qual a figura deve ser rotacionada. Ainda de forma mais elementar, pode-se citar como variação desse recurso o tombamento da figura - que inclusive está presente em programas como Inkscape, MS Paint, entre outros softwares de desenho básico. Em particular, a seguir apresenta-se um exemplo de rotação de um quadrilátero.

$\mathrm{O}$ desenho de formas geométricas de quatro lados - como retângulos e quadrados, por exemplo - é recorrente em diversas demandas de projetos de engenharia. Trata-se de um recurso extremamente comum nos programas de CAD. Ainda, como comando dependente de entradas do usuário, em geral, pode se utilizar diferentes sequências de informações para se obter o quadrilátero. A seguir ilustra-se o caso em que o usuário indica um ponto de referência $p_{r}=\left(x_{r}, y_{r}\right) \mathrm{e}$, posteriormente, a largura $l$ e altura $h$ do objeto bidimensional. Dessa forma, facilmente podem ser obtidos os quatro pontos que definem os vértices do quadrilátero, tal que

$$
\begin{array}{ll}
p_{1}=p_{r} & p_{2}=p_{r}+(l, 0) \\
p_{3}=p_{r}+(l, h) & p_{4}=p_{r}+(0, h)
\end{array}
$$

Assim, a partir dos pontos que definem dois vértices consecutivos é possível obter as arestas $a, b, c$ e $d$ do quadrilátero por $y-y_{i}=m_{i}$ $\left(x-x_{i}\right)$, sendo $m_{i}=\left(y_{i+1}-y_{i}\right) /\left(x_{i+1}-x_{i}\right)$, conforme ilustra a Figura 3. 
Figura 3 - Ilustração do quadrilátero com vértices $p_{i}$ $\mathrm{e}$ arestas (lados) $\mathrm{abcd}$

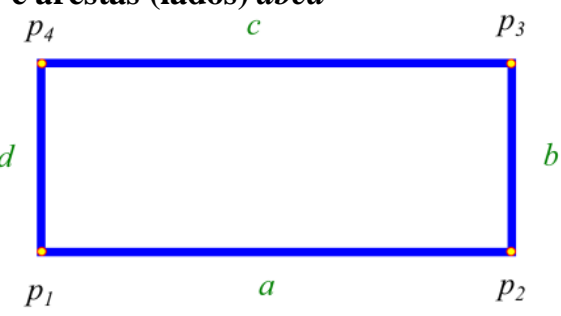

Fonte: acervo dos autores.

Em geral, o referido recurso de rotação de figuras planas disponível nos programas de CAD permite a definição do ponto em torno do qual a figura pode ser rotacionada. Neste trabalho, sem perda de generalidade, apresentase o caso particular de rotação em torno do centro geométrico $p_{c g}$ do quadrilátero, sendo

$p_{c g}=\left(x_{c g}, y_{c g}\right)$ tal que

$x_{c g}=\left(x \mid 2+x_{1}\right) / 2$ e $y_{c g}=\left(y \mid 3+y_{2}\right) / 2$

No entanto, facilmente pode-se definir qualquer outro ponto do plano, inclusive quaisquer dos vértices $p_{i}(i=1, \ldots, 4)$. Assim, para considerar que o ponto $p_{c g}$ define a origem de um sistema de coordenadas locais, em torno do qual se tem a rotação, pode-se empregar a notação vetorial que define o vetor posição de cada um dos pontos com relação à origem do sistema de coordenadas global (sistema $x y$ da Figura 3). Assim, tem-se

$$
\mathbf{p}_{i}=x_{i} \vec{\imath}+y_{i} \vec{\jmath} \quad \text { e } \quad \mathbf{p}_{c g}=x_{c g} \vec{\imath}+y_{c g} \vec{\jmath}
$$

sendo $\vec{\imath}$ e $\vec{\jmath}$ vetores diretores dos eixos $x$ e $y$, respectivamente. Nota-se que, sendo a origem $o$ do sistema global definida em $x=y=0$, cada i-ésimo vetor posição é obtido pelo seguimento $\overline{o p}_{i}=\left(x_{i}-0\right) \vec{\imath}+\left(y_{i}-0\right) \vec{\jmath}$.

A partir dos vetores posição definidos, é possível obter os vetores diferença $p_{i}^{l}$ que, essencialmente, representam os vetores posição de cada $i$-ésimo vértice escritos no sistema de coordenada local ${ }^{4}$ com origem no ponto $p_{c g} \mathrm{em}$ torno do qual a rotação é requerida. Assim, temse

$$
\mathbf{p}_{i}^{l}=\mathbf{p}_{i}+\mathbf{p}_{c g}
$$

Conhecidos os vetores $\mathbf{p}_{i}^{l}$ é possível considerar o operador linear matricial do $\mathbb{R}^{2}$ definido por $\mathbf{T}(\theta)$, sendo $\theta$ o ângulo de rotação, em radianos, no sentido anti-horário

$$
\mathbf{T}(\theta)=\left[\begin{array}{cc}
\cos (\theta) & -\sin (\theta) \\
\sin (\theta) & \cos (\theta)
\end{array}\right]
$$

Finalmente, pode-se obter a nova posição ${ }^{5}$ de cada $i$-ésimo vértice $\mathbf{p}_{i}^{(r o t)}=x_{i}^{(r o t)} \vec{\imath}+y_{i}^{(r o t)} \vec{\jmath}$ da figura rotacionada por

$$
\mathbf{p}_{i}^{(r o t)}=\mathbf{T}(\theta) \mathbf{p}_{i}^{l}+\mathbf{p}_{c g}
$$

Para clareza de entendimento, apresenta-se a seguir um exemplo para uma rotação de $30^{\circ}$ $(\theta=30 \pi / 180)$. Na Figura 4 o quadrilátero original é indicado por linhas tracejadas com vértices destacados por círculos. O ponto $\mathbf{p}_{c g}$, origem do sistema local de coordenadas, é indicado pelo símbolo $\times$, enquanto, finalmente, o objeto rotacionado é representado com vértices triangulares e linhas contínuas definindo suas arestas.

Figura 4 - Quadrilátero rotacionado em $30^{\circ}$ no sentido anti-horário

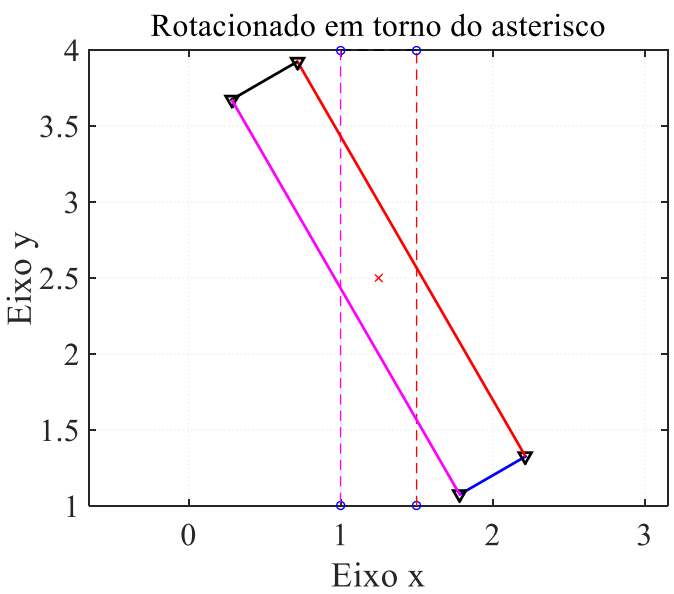

Fonte: acervo dos autores. 


\section{Espelhar objetos}

Um recurso dos programas de CAD amplamente utilizado no desenho técnico é, em geral, traduzido nas versões em português por espelhar (ou mirror, em inglês). Tal recurso, na prática, consiste em representar uma réplica idêntica do objeto através de um plano de referência - que, no sentido figurado, indica um espelho. Assim, de forma análoga ao exemplo da seção anterior, os pontos que definem um objeto - no plano ou no espaço - podem ser rotacionados em torno de um eixo de interesse para, na sequência, definirem a nova posição do objeto, ou seja, o objeto espelhado.

Nesse caso, considerando como exemplo um paralelepípedo regular, cada $i$-ésimo vértice $(i=1, \ldots, 8)$ tem o vetor posição com relação à origem do sistema global de coordenadas definido por $\mathbf{p}_{i}=x_{i} \vec{\imath}+y_{i} \vec{\jmath}+z_{i} \vec{k}$. Para isso, similarmente à seção anterior, é preciso previamente conhecer os oito pontos que os definem, obtendo, por exemplo, um ponto de referência $p_{r}=\left(x_{r}, y_{r}, z_{r}\right)$, dimensões de largura da base $\left(l_{x}\right.$ e $\left.l_{y}\right)$, bem como altura $l_{z}$ do objeto. Tem-se então

$$
\begin{array}{ll}
p_{1}=p_{r} & p_{2}=p_{r}+\left(0, l_{y}, l_{z}\right) \\
p_{3}=p_{r}+\left(0,0, l_{z}\right) & p_{4}=p_{r}+\left(0, l_{y}, 0\right) \\
p_{5}=p_{r}+\left(l_{x}, 0,0\right) & p_{6}=p_{r}+\left(l_{x}, l_{y}, 0\right) \\
p_{7}=p_{r}+\left(l_{x}, 0, l_{z}\right) & p_{8}=p_{r}+\left(l_{x}, l_{y}, l_{z}\right)
\end{array}
$$

Para ilustrar um exemplo de espelhar através do plano $x y$, pode-se considerar o operador linear $\mathbf{T}$, que rotaciona em torno do eixo $x$, definido por

$$
\mathbf{T}=\left[\begin{array}{ccc}
1 & 0 & 0 \\
0 & 1 & 0 \\
0 & 0 & -1
\end{array}\right]
$$

Assim, podem ser obtidos os novos pontos $p_{i}^{n}=\left(x_{i}^{n}, y_{i}^{n}, z_{i}^{n}\right)$ que definem o objeto espelhado considerando a seguinte transformação linear ${ }^{6}$

$$
\mathbf{p}_{i}^{n}=\mathbf{T p}_{i}
$$

Para clareza de entendimento, considera-se o paralelepípedo regular espelhado através do plano $x y$ (ou $z=0$ ) apresentado na Figura 5. Destaca-se em linhas tracejadas a posição original do objeto, cujos vértices são representados pelo símbolo $\circ$, bem como a posição final indicada por linhas contínuas.

Figura 5 - Paralelepípedo espelhado através do plano $z=0$

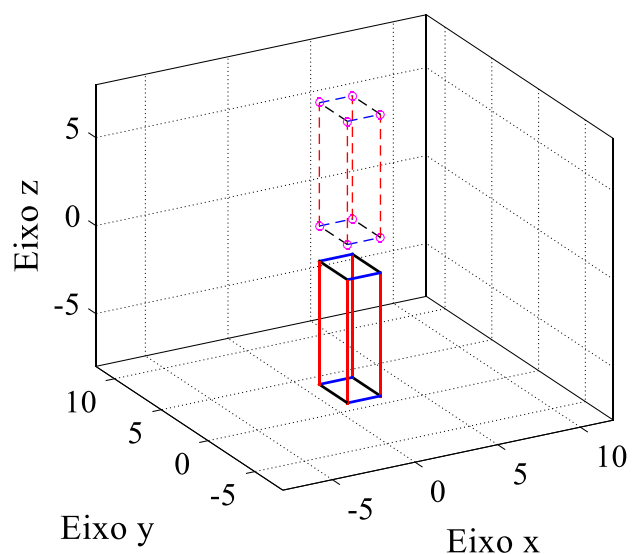

Fonte: acervo dos autores.

\section{Gerar planos de corte}

O desenhista comumente precisa gerar uma visualização da parte interna da peça projetada. Para isso, é necessário gerar planos de corte na peça. Portanto, nesta seção apresenta-se como se pode obter a equação cartesiana de um plano de corte qualquer.

Um plano $\pi$ localizado no sistema de coordenadas espacial $(x, y, z)$ possui a seguinte equação geral

$$
\pi: a x+b y+c z=d
$$

Essa equação pode ser obtida a partir de três pontos não-colineares localizados no espaço. Assim, dados os pontos $A=$ $\left(a_{1}, a_{2}, a_{3}\right), B=\left(b_{1}, b_{2}, b_{3}\right)$ e $C=\left(c_{1}, c_{2}, c_{3}\right)$, obtém-se dois vetores diretores $\overrightarrow{A B}$ e $\overrightarrow{A C}$ paralelos ao plano que são dados por

$$
\overrightarrow{A B}=\left(x_{1}, y_{1}, z_{1}\right) \text { e } \overrightarrow{A C}=\left(x_{2}, y_{2}, z_{2}\right)
$$

sendo 


$$
\begin{aligned}
& x_{1}=b_{1}-a_{1}, y_{1}=b_{2}-a_{2}, z_{1}=b_{3}-a_{3} \\
& x_{2}=c_{1}-a_{1}, y_{2}=c_{2}-a_{2}, z_{2}=c_{3}-a_{3}
\end{aligned}
$$

Então, o vetor normal ao plano procurado, representado por $\vec{N}$ é obtido através do produto vetorial $\vec{N}=\overrightarrow{A B} \times \overrightarrow{A C}$. Visto que os pontos $A$, $B$ e $C$ não são colineares, o resultado desse produto vetorial é não nulo, e dado por $\vec{N}=$ $\left(n_{1}, n_{2}, n_{3}\right) \quad$ com $n_{1}=y_{1} z_{2}-z_{1} y_{2}, n_{2}=$ $z_{1} x_{2}-x_{1} z_{2}$ e $n_{3}=x_{1} y_{2}-y_{1} x_{2}$.

A partir do vetor normal, e escolhendo qualquer um dos três pontos (por exemplo ponto $A$ ), pode-se obter o conjunto de pontos $P=(x, y, z)$ que gera o plano $\pi$, resolvendo o produto escalar $\vec{N} \cdot \overrightarrow{A P}=0$. Assim, tem-se a seguinte equação cartesiana

$$
\pi: \quad n_{1} x+n_{2} y+n_{3} z+d=0
$$

sendo $d=-n_{1} a_{1}-n_{2} a_{2}-n_{3} a_{3}$.

A Figura 6 mostra o plano $\pi$ gerado para o corte de uma superfície esférica considerando três pontos, $A, B$ e $C$, escolhidos arbitrariamente na superfície a ser cortada. Esses pontos devem satisfazer a seguinte equação da superfície $S$ da esfera:

$$
S:\left(x-x_{0}\right)^{2}+\left(y-y_{0}\right)^{2}+\left(z-z_{0}\right)^{2}=r^{2}
$$

sendo $r$ o raio da esfera e $\left(x_{0}, y_{0}, z_{0}\right)$ as coordenadas do centro da esfera.

A Equação 13 pode ser escrita na seguinte forma paramétrica

$$
\begin{gathered}
x=x_{0}+r \cos \theta \sin \emptyset \\
y=y_{0}+r \sin \theta \sin \emptyset \\
z=z_{0}+r \cos \emptyset
\end{gathered}
$$

sendo o intervalo angular dado por $0 \leq \theta \leq$ $2 \pi$ e $0 \leq \emptyset \leq \pi$. Nota-se que, a partir da Equação 14, para escolher pontos contidos na superfície esférica, basta escolher posições angulares $(\theta, \varnothing)$.
Figura 6 - Plano de corte sobre uma esfera de raio $r=1$, com centro em $x_{0}=y_{0}=z_{0}=0$ e, plano gerado a partir dos pontos $A\left(-30^{\circ},-100^{\circ}\right), B\left(0^{\circ}, 30^{\circ}\right)$ e $C\left(30^{\circ},-\right.$ $100^{\circ}$ )

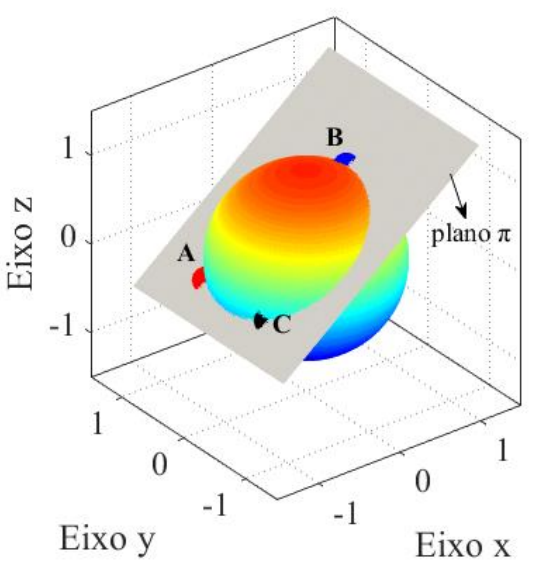

Fonte: acervo dos autores.

A Figura 7 mostra a vista ortogonal xy (observador na direção y) para a superfície esférica e plana mostrado na Figura 6. Nesse caso, a projeção do plano é uma reta e a projeção da esfera é um círculo de raio $r$. Notase que a reta é perpendicular ao vetor normal $\vec{N}$, tem ângulo de inclinação $\alpha \mathrm{e}$ as intersecções são dadas pelos pontos $P$ e $Q$.

\section{Figura 7 - Vista ortogonal xz para o plano e esfera} mostrados na Figura 6

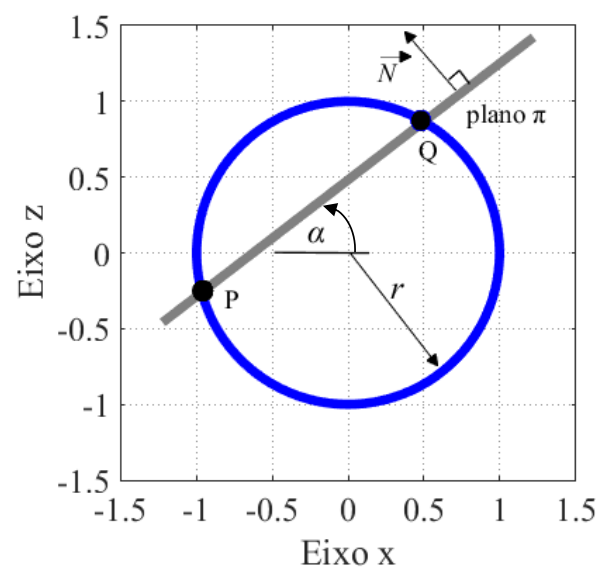

Fonte: acervo dos autores.

\section{Vista ortogonal}

Essa seção mostra como se obter a vista ortogonal no plano cartesiano xy (observador na direção z) utilizando a curva de intersecção gerada pelo plano e esfera mostrados na Figura 6.

Da equação do plano (Equação 12), tem-se 


$$
z=\frac{1}{n_{3}}\left(-n_{1} x-n_{2} y-d\right)
$$

Substituindo-se a Equação 15 na equação da esfera (Equação 13), para origem em $x_{0}=y_{0}=$ $z_{0}=0$, resulta em uma equação escrita na forma

$$
A x^{2}+B x y+C x^{2}+D x+E y+F=0
$$

sendo

$$
\begin{gathered}
A=1+\left(\frac{n_{1}}{n_{3}}\right)^{2} ; B=\frac{2 n_{1} n_{2}}{n_{3}{ }^{2}} ; \\
C=1+\left(\frac{n_{2}}{n_{3}}\right)^{2} ; D=\frac{2 n_{1} d}{n_{3}{ }^{2}} ; \\
E=\frac{2 n_{2} d}{n_{3}{ }^{2}} \quad ; F=\left(\frac{d}{n_{3}}\right)^{2}-R^{2}
\end{gathered}
$$

A Equação 16 define uma elipse se $B^{2}<$ $4 A C$ e, no caso de $A=C, A \neq 0$ e $B=0$, corresponde ao círculo. Essa equação pode ser escrita na seguinte forma paramétrica

$$
\begin{aligned}
& x=h+a \cos \theta \cos \tau-b \sin \theta \sin \tau \\
& y=k+a \cos \theta \sin \tau+b \sin \theta \cos \tau
\end{aligned}
$$

sendo $h$ e $k$ as coordenadas do centro da elipse, $a$ e $b$ os semi-eixos da elipse e $\tau$ o ângulo de rotação da elipse, que são dados por

$$
\begin{gathered}
h=(B E-2 C D) /\left(4 A C-B^{2}\right) \\
k=(B D-2 A E) /\left(4 A C-B^{2}\right) \\
a=\sqrt{-\operatorname{det}\left(M_{0}\right) /(\operatorname{det}(M)) \lambda_{1}} \\
b=\sqrt{-\operatorname{det}\left(M_{0}\right) /(\operatorname{det}(M)) \lambda_{2}} \\
\tau=\operatorname{arccot}\left(\frac{A-C}{B}\right) / 2
\end{gathered}
$$

sendo $\operatorname{det}($.$) o determinante das matrizes$

$$
M_{0}=\left[\begin{array}{ccc}
F & D / 2 & E / 2 \\
D / 2 & A & B / 2 \\
E / 2 & B / 2 & C
\end{array}\right],
$$

$M=\left[\begin{array}{cc}A & B / 2 \\ B / 2 & C\end{array}\right]$ e $\lambda_{1}, \lambda_{2}$ os autovalores da matriz $M$ ordenados tal que $\left|\lambda_{1}-A\right| \leq \mid \lambda_{1}-$ $C \mid$ ou $\left|\lambda_{2}-C\right| \leq\left|\lambda_{2}-A\right|$.

Portanto, utilizando as Equações 17 e 18 para o plano e esfera mostrados na seção anterior (ver Figura 6) se obtém a elipse mostrada na Figura 8, que representa a curva de interseção das superfícies e a vista ortogonal no plano cartesiano xy.

Figura 8 - Vista ortogonal xy da intersecção do plano e esfera mostrados na Figura 6

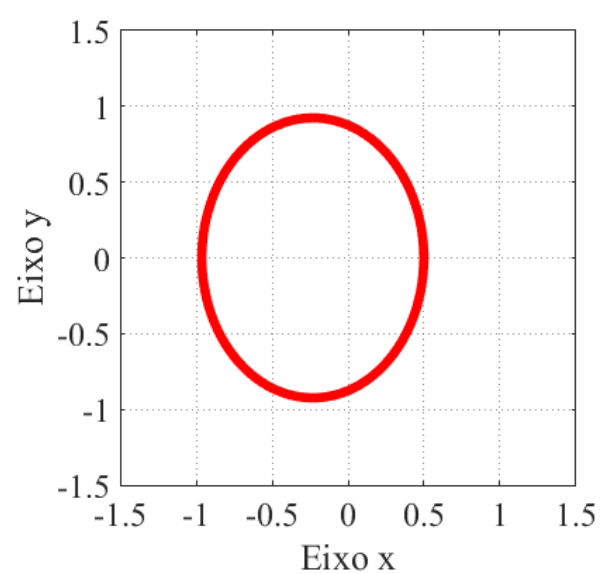

Fonte: acervo dos autores.

\section{Vista auxiliar}

Outra vista comumente observada pelo desenhista é a vista auxiliar. Considerando a Figura 6, uma vista auxiliar pode ser representada pela curva de intersecção das superfícies (plano e esfera) quando o observador está na direção do vetor normal $\vec{N}$ do plano. Nesse caso, a curva é um círculo com centro $c$ conforme mostrado a seguir.

Considera-se uma esfera com centro em $c_{0}\left(x_{0}, y_{0}, z_{0}\right)$ e raio $r>0$, e um plano $\pi$ dado pela Equação 12 com vetor normal $\vec{N}$ e sendo $p(x, y, z)$ um ponto qualquer de $\pi$, conforme mostra a Figura 9.

\section{Figura 9 - Ilustração para obter o círculo da} intersecção entre plano e esfera

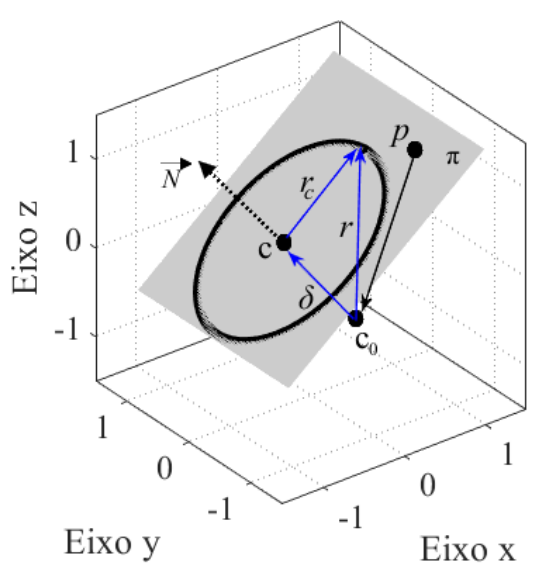

Fonte: acervo dos autores. 
A menor distância $\delta$ do centro co da esfera ao plano é obtida utilizando a propriedade do produto escalar dos vetores $\overrightarrow{p c_{0}} \cdot \vec{N}=$ $\left|\overrightarrow{p c_{0}}\right||\vec{N}| \cos (\varphi)$, sendo $\varphi$ o ângulo entre os vetores. Assim, para a condição de menor distância temos que $\left|\overrightarrow{p c_{0}}\right|=\delta$ e o vetor $\overrightarrow{p c_{0}}$ é paralelo ao vetor $\vec{N}$, isto é, $\varphi=0$. Assim, temse

$$
\delta=\frac{\overrightarrow{p c_{0}} \cdot \vec{N}}{|\vec{N}|}=\frac{\left(n_{1} x_{0}+n_{2} y_{0}+n_{3} z_{0}-d\right)}{\sqrt{n_{1}^{2}+n_{2}^{2}+n_{3}^{2}}}
$$

E o centro $c\left(x_{\mathrm{c}}, y_{\mathrm{c},,}, z_{\mathrm{c}}\right)$ e o raio $r_{\mathrm{c}}$ são dados por

$c=c_{0}+\delta \frac{\left(n_{1}, n_{2}, n_{3}\right)}{\sqrt{n_{1}^{2}+n_{2}^{2}+n_{3}^{2}}}$

$r_{c}=\sqrt{r^{2}-\delta^{2}}$.

E a equação paramétrica para o círculo é dada por

$$
\begin{aligned}
& x_{\text {circ }}=x_{c}+r_{c} \cos \theta \\
& y_{\text {circ }}=y_{c}+r_{c} \sin \theta
\end{aligned}
$$

Finalmente, utilizando as Equações 20 e 21 para o plano e esfera mostrados na Figura 6, obtém-se o círculo mostrado na Figura 10, que representa a vista auxiliar quando o observador está na direção do vetor normal do plano.

Figura 10 - Vista auxiliar para a intersecção do plano e esfera mostrados na Figura 6

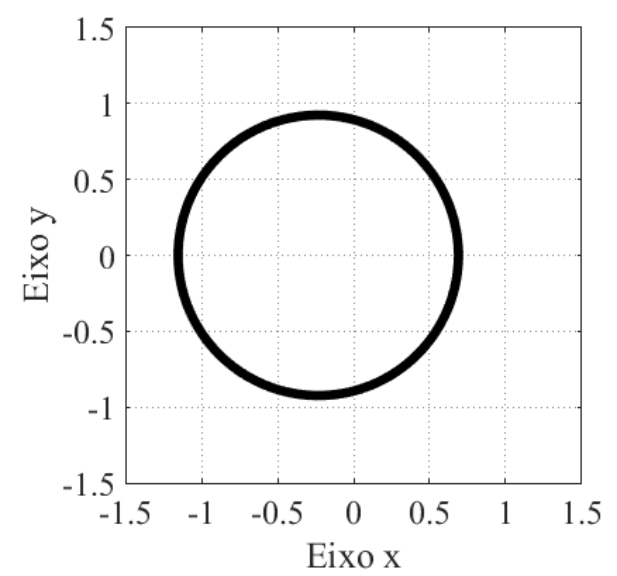

Fonte: acervo dos autores.

\section{CONCLUSÕES}

Neste artigo apresenta-se uma reflexão sobre oportunidades de aprendizado do desenho técnico para cursos de engenharia. As ideias são introduzidas no contexto da engenharia mecânica e podem naturalmente ser estendidas a cursos afins.

Apresenta-se uma visão do ensino do desenho técnico mecânico, que essencialmente destaca a oportunidade de integrar elementos de matemática e computação às aulas de prática de desenhos com execução via CAD. A integração proposta não compreende adicionar o ensino de conteúdos de álgebra, geometria analítica, computação, entre outros, nas ementas de cursos de desenho técnico. Entende-se que o pré-requisito básico para exercitar este tipo de integração é naturalmente atendido para os casos de educandos que previamente cursaram, ou estejam cursando, essas demais disciplinas típicas do curso de Engenharia Mecânica, enquanto participantes do curso de desenho técnico mecânico. Assim, entende-se que a prática do $\mathrm{CAD}$ com tal integração pode, por consequência, também contribuir nos aprendizados de elementos da álgebra e geometria analítica, entre outras, uma vez que permite interessante associação geométrica com os elementos do desenho. Neste sentido, o ensino integrado tem interessante desdobramento na formação dos educandos.

Em particular, os exemplos apresentados neste artigo foram implementados em ambiente OCTAVE - que podem ser facilmente reproduzidos por educandos e educadores. Por ser software livre, de linguagem interpretada, de fácil acesso e multiplataforma, considera-se que o OCTAVE é software conveniente para exercitar essas implementações computacionais. No entanto, diversos outros podem ser empregados, como por exemplo o Scilab, o Python e o Matlab.

A reflexão apresentada neste trabalho se dá no contexto de educando dos primeiros semestres de cursos de Engenharia Mecânica. No entanto, entende-se que resultados equivalentes podem ser obtidos para aqueles em fase final de formação. 


\section{REFERÊNCIAS}

GARDNER, H. Frames of Mind: The Theory of Multiple Intelligences, Basic Books, $3^{\text {a }}$ edition, 2011.

MONNERAT, L. P. Uma abordagem para a melhoria do processo de ensinoaprendizagem em desenho técnico utilizando métodos e técnicas da computação. Dissertação (Mestrado) Universidade Federal de Viçosa, Pós Graduação em Ciência da Computação, 2012.

PEREIRA, T. R. D. S., et al. O ensino do desenho técnico no curso de engenharia: uma proposta pedagógica. Anais... XXXIX Congresso Brasileiro de Educação em Engenharia COBENGE, 2011.
SILVA, F. V. P. Um sistema hipermídia no ensino de desenho técnico-mecânico. Dissertação (Mestrado) - Universidade Estadual do Ceará, Centro Federal de Educação Tecnológica, Mestrado Integrado Profissionalizante em Computação, 2003.

SILVA, J. C. Aprendizagem mediada por computador: uma proposta para desenho técnico mecânico. Tese (Doutorado) Universidade Federal de Santa Catarina, PósGraduação em Engenharia de Produção, 2001.

TRINDADE, B. Ambiente híbrido para a aprendizagem dos fundamentos de desenho técnico para as engenharias. Tese (Doutorado) - Universidade Federal de Santa Catarina, Pós-Graduação em Engenharia de Produção, 2002.

\section{DADOS BIOGRÁFICOS DOS AUTORES}
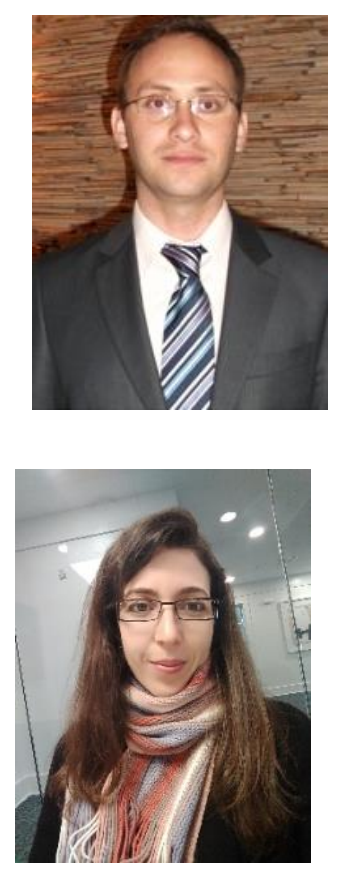

Douglas Domingues Bueno - Possui graduação e mestrado em Engenharia Mecânica (2006 e 2007) pela Faculdade de Engenharia de Ilha Solteira (Feis, UNESP) e doutorado em Engenharia Aeronáutica (2014) pelo ITA. Realizou estágio de pós-doutorado na Duke University (EUA, 2019). Atuou nas indústrias automobilística (PSA Peugeot Citröen) e aeronáutica (Embraer SA) como engenheiro de desenvolvimento de produto. Atualmente é professor na Feis UNESP, lecionando também desenho técnico mecânico. Tem experiência no ensino de engenharia, com foco de pesquisas em aeroelasticidade.

Camila Gianini Gonsalez Bueno - Possui graduação, mestrado (2008 e 2012) e doutorado (2019) em Engenharia Mecânica pela Faculdade de Engenharia de Ilha Solteira (Feis, UNESP). Atuou nas indústrias automobilística (ZF Sachs e General Motors) e aeronáutica (Embraer SA) como engenheira de desenvolvimento de produto. Atualmente é pesquisadora em pós-doutorado na Feis UNESP. Tem experiência em propagação de ondas em estruturas e monitoramento da integridade estrutural. 


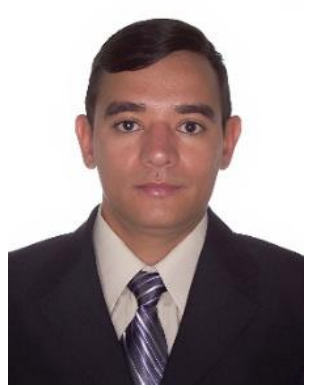

Rodrigo Borges Santos - Possui graduação em Engenharia Mecânica (2005), mestrado (2008) e doutorado (2018) pela Faculdade de Engenharia de Ilha Solteira (Feis, UNESP). Atuou na área de gestão da qualidade da Moto Honda da Amazônia Ltda (2008-2010) e como engenheiro de produto na área de Acústica e Vibração na PSA Peugeot Citroën do Brasil Automóveis (2010-2015). Atualmente é professor Adjunto no departamento de Engenharia Mecânica da Universidade Federal da Grande Dourados - UFGD. Tem experiência na área de mecânica dos sólidos, atuando principalmente em controle de vibrações e propagação de ondas em estruturas periódicas. 